\title{
A classification of the face numbers of Buchsbaum simplicial posets
}

\author{
Jonathan Browder ${ }^{1} \|^{*}$ and Steven Klee $\|^{*}$ \\ ${ }^{1}$ Aalto University, Department of Mathematics, Helsinki, Finland \\ ${ }^{2}$ Seattle University, Department of Mathematics, Seattle, WA, 98122, USA
}

\begin{abstract}
The family of Buchsbaum simplicial posets generalizes the family of simplicial cell manifolds. The $h^{\prime}-$ vector of a simplicial complex or simplicial poset encodes the combinatorial and topological data of its face numbers and the reduced Betti numbers of its geometric realization. Novik and Swartz showed that the $h^{\prime}$-vector of a Buchsbaum simplicial poset satisfies certain simple inequalities. In this paper we show that these necessary conditions are in fact sufficient to characterize the $h^{\prime}$-vectors of Buchsbaum simplicial posets with prescribed Betti numbers.

Resumé. La famille des ordres simpliciaux de Buchsbaum est une généralisation de la famille des variétés cellules simpliciaux. Les informations combinatoriques et topologiques des nombres- $f$ et des nombres réduits de Betti de la réalisation géométrique d'un complex simplicial ou un ordre simplicial sont encodées dans la vecteur- $h^{\prime}$. Novik et Swartz ont montrés que la vecteur- $h^{\prime}$ d'un ordre simplicial de Buchsbaum se remplit à certaines inégalitiés simples. Dans cet article, nous démontrons que ces conditions nécessaires sont, en effet, suffisante pour classer les vecteurs- $h^{\prime}$ des ordres simpliciaux de Buchsbaum avec des nombres de Betti prescrits.
\end{abstract}

Keywords: Simplicial complex; simplicial poset; $f$-vector; $h$-vector; Cohen-Macaulay; Buchsbaum

\section{Introduction}

A (finite) simplicial poset is a poset with a unique minimal element $\hat{0}$ in which each interval $[\hat{0}, \tau]$ is isomorphic to a Boolean lattice. Such a poset is naturally graded by declaring that an element $\tau$ has rank $k$ if the interval $[\hat{0}, \tau]$ is isomorphic to the Boolean lattice of rank $k$.

Any simplicial poset $P$ can be naturally identified with a topological space $|P|$ by associating a $(k-1)$ dimensional simplex to each rank $k$ element of $P$ in such a way that the order relation on $P$ dictates the containment of faces in $|P|$. As such, simplicial posets are also typically called simplicial cell complexes. The primary difference between a simplicial poset and a simplicial complex is that any pair of faces in a simplicial complex intersect along a single (possibly empty) face of their boundaries; whereas a pair of faces in a simplicial poset can intersect along any sub complex of their boundaries.

We are particularly interested in the families of Cohen-Macaulay and Buchsbaum simplicial posets. The former family of simplicial posets is an algebraic generalization of the families of simplicial cell balls and

*Email: jonathan.browder@alto.fi

†Email: klees@seattleu.edu

1365-8050 @ 2014 Discrete Mathematics and Theoretical Computer Science (DMTCS), Nancy, France 
simplicial cell spheres (i.e., simplicial cell complexes that triangulate a ball/sphere of some dimension) and the latter family generalizes the family of simplicial cell manifolds (with or without boundary).

The most fundamental combinatorial invariant of a simplicial poset $P$ of rank $d$ is its $f$-vector, which is denoted $f(P)=\left(f_{-1}(P), f_{0}(P), f_{1}(P), \ldots, f_{d-1}(P)\right)$, and the $f$-numbers $f_{i}(P)$ count the number of $i$-dimensional faces in $P$. The primary question that arises in the study of simplicial complexes or posets is to fix some family of complexes or posets (for example, Cohen-Macaulay or Buchsbaum complexes/posets or, alternatively, the collection of all simplicial complexes/posets that triangulate a given topological space) and determine necessary and sufficient conditions on the collection of $f$-vectors that can arise from triangulations in that family.

For the purposes of answering such questions, it is often more convenient to study the $h$-vector of a simplicial poset, which is denoted $h(P)=\left(h_{0}(P), h_{1}(P), \ldots, h_{d}(P)\right)$, and whose entries are called the $h$-numbers of $P$. The $h$-numbers of $P$ are defined as a certain integer transformation of its $f$-numbers, and knowing the $f$-numbers of $P$ is equivalent to knowing its $h$-numbers.

For example, in the case that $P$ is Cohen-Macaulay, the $h$-numbers of $P$ count the graded dimensions of the quotient of the face ring of $P$ by a linear system of parameters. Stanley (1977) gave a complete characterization of the $h$-numbers of Cohen-Macaulay simplicial complexes, and later Stanley (1991) gave a complete characterization of the $h$-numbers of Cohen-Macaulay simplicial posets.

A recurring theme in this area is that such characterization theorems for simplicial posets are often much simpler than their counterparts for simplicial complexes; or in other cases, characterizations are known for certain families of simplicial posets that are still unsolved for those same families of simplicial complexes. For example Stanley (1991) and Masuda (2005) gave a complete characterization of the $h$ vectors of simplicial cell spheres, while it is still unknown whether or not the conditions of the $g$-theorem of Stanley (1980) and Billera and Lee (1981), which characterizes the family of $h$-vectors of simplicial polytopes, continues to hold for arbitrary simplicial spheres.

Similarly, Novik and Swartz (2009) established necessary conditions on both the $h$-numbers of Buchsbaum simplicial complexes and the $h$-numbers of Buchsbaum simplicial posets that are natural generalizations of the formulas that appear in the characterizations of $h$-vectors of Cohen-Macaulay simplicial complexes and posets. As in the Cohen-Macaulay realm, the conditions they determine for the family of Buchsbaum simplicial complexes are nonlinear inequalities in the style of the Macaulay (1972) inequalities that bound the relative growth of the entries of an $M$-sequence, while the inequalities for Buchsbaum simplicial posets are simple linear lower bounds.

The remainder of the paper is structured as follows. In Section 2 we outline the history of this problem, along with the relevant background definitions on simplicial complexes and simplicial posets. In Section 3. we will present our contribution to this problem, which shows that the necessary conditions imposed by Novik and Swartz (2009) are in fact sufficient to characterize the $h$-vectors of Buchsbaum simplicial posets with prescribed homology groups. We will omit most of the proofs in this section, and we will only sketch the proof of our main result. This abstract is a summary of the results in Browder and Klee (2013), and refer to that paper for the complete details of our proofs.

\section{History and Background}

\subsection{Simplicial complexes and simplicial posets}

A simplicial complex, $\Delta$, on vertex set $V=V(\Delta)$ is a collection of subsets $\tau \subseteq V$, called faces, with the property that if $\tau \in \Delta$ and $\sigma \subseteq \tau$, then $\sigma \in \Delta$. The dimension of a face $\tau \in \Delta \operatorname{is} \operatorname{dim}(\tau):=|\tau|-1$, and 
the dimension of $\Delta$ is $\operatorname{dim}(\Delta):=\max \{\operatorname{dim}(\tau): \tau \in \Delta\}$. The link of a face $\tau \in \Delta$ is

$$
\operatorname{lk}_{\Delta}(\tau)=\{\gamma \in \Delta: \gamma \cap \tau=\emptyset, \gamma \cup \tau \in \Delta\}
$$

The face poset of a simplicial complex is the poset of faces of $\Delta$ ordered by inclusion. The empty set is clearly the unique minimal element of the face poset of any (nonempty) simplicial complex. Moreover, the face poset is naturally graded by $\operatorname{rk}(\tau)=|\tau|$; and since any face in a simplicial complex is determined by its vertices, any interval $[\sigma, \tau]:=\{\rho \in \Delta: \sigma \subseteq \rho \subseteq \tau\}$ in the face poset is a Boolean lattice of rank $\operatorname{rk}(\tau)-\operatorname{rk}(\sigma)$.

More generally, a simplicial poset is a poset $P$ with a unique minimal element $\hat{0}$ such that any interval $[\hat{0}, \tau]$ in $P$ is a Boolean lattice. The face poset of a simplicial complex is a simplicial poset, and hence simplicial posets serve as natural generalizations of simplicial complexes. Many of the commonly-studied combinatorial and topological properties of simplicial complexes translate directly into corresponding properties of simplicial posets. We refer to Stanley's book Stanley (1996) for further background information.

As with simplicial complexes, a simplicial poset is naturally graded by declaring that $\operatorname{rk}(\tau)=r$ if $[\hat{0}, \tau]$ is a Boolean lattice of rank $r$. For any simplicial poset $P$, there is a regular CW-complex $|P|$, called the geometric realization of $P$, whose face poset is $P$. The closed cells of $P$ are simplices with every pair of cells intersecting along a (possibly empty) subcomplex of their boundaries (as opposed to a single face, as is the case for simplicial complexes); such a CW-complex is called a simplicial cell complex. Hereafter we will use the terminology of simplicial poset $P$ and that of its associated simplicial cell complex $|P|$ interchangeably, so a rank $r$ element of $P$ is an $(r-1)$-dimensional face, and the dimension of $P$ is $\operatorname{dim}(P)=\operatorname{dim}(|P|)=\operatorname{rk}(P)-1$. In particular, following the poset terminology, we refer to the rankone elements of a simplicial poset as atoms; the atoms of $P$ correspond to the vertices of $|P|$. We say $P$ is pure if all of its maximal faces have the same dimension.

The order complex of a simplicial poset $P$ is the simplicial complex $\Delta(P)$ whose vertices are the elements of $P-\hat{0}$, and whose faces are chains of the form $\tau_{0}<\tau_{1}<\cdots<\tau_{r}$. Topologically, $|\Delta(P)|$ is the barycentric subdivision of $|P|$.

The link of a face $\sigma$ in a simplicial poset $P$ is

$$
\operatorname{lk}_{P}(\sigma)=\{\tau \in P: \tau \geq \sigma\} .
$$

It is easy to see that $\operatorname{lk}_{P}(\sigma)$ is a simplicial poset whose unique minimal element is $\sigma$. If $P$ is the face poset of a simplicial complex, $\operatorname{lk}_{P}(\sigma)$ is the face poset of the link of $\sigma$ in $\Delta$.

The most natural combinatorial invariant of a finite $(d-1)$-dimensional simplicial poset is its $f$-vector, $f(P):=\left(f_{-1}(P), f_{0}(P), \ldots, f_{d-1}(P)\right)$, where the $f$-numbers $f_{i}(P)$ count the number of $i$-dimensional faces in $P$. Often it is more natural to study a certain integer transformation of the $f$-vector called the $h$-vector, $h(P):=\left(h_{0}(P), h_{1}(P), \ldots, h_{d}(P)\right)$ whose entries, the $h$-numbers of $P$, are defined by

$$
h_{j}(P)=\sum_{i=0}^{j}(-1)^{j-i}\left(\begin{array}{l}
d-i \\
d-j
\end{array}\right) f_{i-1}(P) .
$$

For any $(d-1)$-dimensional simplicial poset $P, h_{0}(P)=1$ and $h_{d}(P)=(-1)^{d-1} \widetilde{\chi}(P)$, where $\tilde{\chi}(P)$ denotes the reduced Euler characteristic of $P$. Since the Euler characteristic of $P$ is inherently related to 
both the combinatorial and topological structure of $P$, we will also be interested in studying the (reduced) Betti numbers of $P$ (over a field $\mathbf{k}$ ), which are defined as $\widetilde{\beta}_{i}(P)=\widetilde{\beta}_{i}(P ; \mathbf{k}):=\operatorname{dim}_{\mathbf{k}} \widetilde{H}_{i}(P ; \mathbf{k})$.

The primary reason for studying $h$-numbers instead of $f$-numbers is that they arise naturally when studying the face ring of a simplicial poset. We will not define the face ring here since the properties we are interested in studying can be defined equivalently in terms of topological information. We refer to Stanley's book Stanley (1996) for further information on the algebraic properties of face rings.

We will be interested in studying two families of simplicial posets known as Cohen-Macaulay simplicial posets and Buchsbaum simplicial posets. For simplicial complexes, the property of being CohenMacaulay or Buchsbaum is defined as an algebraic condition on the face ring: a simplicial complex $\Delta$ is Cohen-Macaulay (or Buchsbaum) if and only if its face ring is Cohen-Macaulay (or Buchsbaum). A simplicial poset $P$ is Cohen-Macaulay (or Buchsbaum) if and only if its order complex is Cohen-Macaulay (or Buchsbaum). Reisner (1976) showed that the Cohen-Macaulay property for simplicial complexes may be viewed as a topological condition on the geometric realization of a simplicial complex, rather than an algebraic condition on its face ring. Reisner's condition was later generalized to Buchsbaum complexes by Schenzel (1981). We summarize these results in the following theorem, which provides an equivalent definition of Cohen-Macaulay and Buchsbaum simplicial complexes.

Theorem 2.1 $A(d-1)$-dimensional simplicial complex $\Delta$ is Cohen-Macaulay (over a field $\mathbf{k}$ ) if and only if $\widetilde{H}_{i}\left(\mathrm{lk}_{\Delta}(\tau) ; \mathbf{k}\right)=0$, for all faces $\tau \in \Delta$ (including $\tau=\emptyset$ ) and all $i<\operatorname{dim}\left(\mathrm{k}_{\Delta}(\tau)\right)$. The complex $\Delta$ is Buchsbaum (over $\mathbf{k}$ ) if and only if it is pure and the link of each of its vertices is Cohen-Macaulay (over k).

\subsection{Classifications of families of $f$-vectors}

Our goal in this paper is to classify the face vectors of Buchsbaum simplicial posets. We will begin with a brief survey of known results for both simplicial complexes and simplicial posets. As we will see, the classifications that arise for simplicial posets are significantly less restrictive than the analogous results for simplicial complexes.

The first, and most natural question that one can ask is for a classification of which integer vectors can arise as the $f$-vector of some simplicial complex or poset. Kruskal (1963) and Katona (1968) gave a complete classification of $f$-vectors of simplicial complexes, by establishing nonlinear inequalities that bound the relative growth of successive $f$-numbers. In contrast, Stanley (1991) showed that the only condition that is required on the $f$-numbers of a simplicial poset of rank $d$ is that $f_{i-1} \geq\left(\begin{array}{l}d \\ i\end{array}\right)$ : that is to say that such a simplicial poset must contain a face of dimension $d-1$, which in turn contains $\left(\begin{array}{l}d \\ i\end{array}\right)$ faces of dimension $i$; but there are no further restrictions on the $f$-numbers.

Next, we turn our attention to the family of Cohen-Macaulay simplicial complexes and posets. In general, the $h$-numbers of a simplicial complex or poset may be negative; but, in the Cohen-Macaulay case, Stanley (1977. 1991) showed that the $h$-numbers arise naturally as the graded dimensions of the quotient of the face ring by a linear system of parameters. As such, the $h$-numbers of a simplicial complex or poset are nonnegative. Stanley (1977) showed that an integer vector $\mathbf{h} \in \mathbb{Z}^{d+1}$ is the $h$-vector of a Cohen-Macaulay simplicial complex of dimension $d-1$ if and only $\mathbf{h}$ is an $M$-sequence. This means that in addition to being nonnegative, the entries of $\mathbf{h}$ must satisfy the nonlinear Macaulay (1972) inequalities that bound their relative growth.

As a parallel to the classification of $f$-vectors of arbitrary simplicial complexes/posets, Stanley (1991) 
showed that the conditions that classify $h$-vectors of Cohen-Macaulay simplicial posets are far less restrictive than those that classify their counterparts for simplicial complexes.

Theorem 2.2 Stanley, 1991, Theorem 3.10) A vector $\mathbf{h}=\left(h_{0}, h_{1}, \ldots, h_{d}\right) \in \mathbb{Z}^{d+1}$ is the h-vector of a Cohen-Macaulay simplicial poset of rank $d$ if and only if $(1) h_{0}=1$, and (2) $h_{j} \geq 0$ for all $j$.

Schenzel (1981) (for simplicial complexes) and Novik and Swartz (2009) (for simplicial posets) showed that if $P$ is a $(d-1)$-dimensional Buchsbaum simplicial poset, then the graded dimensions of the quotient of the face ring by a linear system of parameters, which we denote by $h_{j}^{\prime}(P)$, are given by the following formula:

$$
h_{j}^{\prime}(P):=h_{j}(P)+\left(\begin{array}{l}
d \\
j
\end{array}\right) \sum_{i=0}^{j-1}(-1)^{j-i-1} \tilde{\beta}_{i-1}(|P| ; \mathbf{k}) .
$$

We call these numbers $h_{j}^{\prime}(P)$ the $h^{\prime}$-numbers of $P$. The above formula shows that the $h^{\prime}$-numbers may also be defined completely in terms of topological and combinatorial information. In particular, Theorem 2.1 implies that every Cohen-Macaulay simplicial poset is also a Buchsbaum simplicial poset, and that the homology groups of a Cohen-Macaulay simplicial poset vanish in codimension-one and higher. Thus, by Equation (2.2), the $h^{\prime}$-numbers of a Cohen-Macaulay simplicial poset agree with its $h$-numbers, and the $h^{\prime}$-numbers of a Buchsbaum simplicial poset are the appropriate generalization of the $h$-numbers of a Cohen-Macaulay simplicial poset for both algebraic and topological reasons.

For Buchsbaum simplicial posets, Novik and Swartz (2009) proved a result that is stronger than the obvious nonnegativity of the $h^{\prime}$-numbers.

Theorem 2.3 ((Novik and Swartz, 2009. Theorem 6.4)) Let P be a Buchsbaum simplicial poset of rank d. Then

$$
h_{j}^{\prime}(P) \geq\left(\begin{array}{l}
d \\
j
\end{array}\right) \tilde{\beta}_{j-1}(|P|) .
$$

In the case that $P$ is Cohen-Macaulay, we have already seen that $h_{j}^{\prime}(P)=h_{j}(P)$ for all $j$; and since $|P|$ can only have nonvanishing homology only in its top degree, the quantities on the right hand side of Equation 2.3 are all zero. Thus Theorem 2.3 is a natural generalization of the result that the $h$-numbers of a Cohen-Macaulay simplicial poset are nonnegative.

Novik and Swartz (2009) went on to ask if the inequality in Theorem 2.3 is sufficient to characterize the $h^{\prime}$-numbers of Buchsbaum simplicial posets with prescribed Betti numbers.

Question 2.4 (Novik and Swartz, 2009, Question 7.4) Are the following conditions sufficient to characterize the $h^{\prime}$-vectors of Buchsbaum simplicial posets with prescribed Betti numbers?

1. $h_{0}^{\prime}=1$,

2. $h_{j}^{\prime} \geq\left(\begin{array}{c}d \\ j\end{array}\right) \tilde{\beta}_{j}$ for all $1 \leq j \leq d-1$, and

3. $h_{d}^{\prime}=\tilde{\beta}_{d-1}$.

The first and third conditions are included because they necessarily hold for Buchsbaum simplicial posets. The first condition is obvious, and the third condition follows from the formula (2.2) in the case that $j=d$, together with the fact that $h_{d}=(-1)^{d-1} \tilde{\chi}(P)=\sum(-1)^{d-i-1} \tilde{\beta}_{i}(P)$ for any simplicial poset. 


\section{$3 h^{\prime}$-vectors of Buchsbaum posets}

Our goal for the remainder of this paper is to answer Question 2.4 in the affirmative in the following theorem.

Theorem 3.1 Let $\beta_{0}, \beta_{1}, \ldots, \beta_{d-1}, h_{0}^{\prime}, h_{1}^{\prime}, \ldots, h_{d}^{\prime}$ be non-negative integers. Then there is a Buchsbaum simplicial poset $P$ of rank $d$ with $h_{i}^{\prime}(P)=h_{i}$ and $\tilde{\beta}_{i}(P)=\beta_{i}$ if and only if $h_{0}^{\prime}=1, h_{d}^{\prime}=\beta_{d-1}$ and for $j=1,2, \ldots, d-1, h_{j}^{\prime} \geq\left(\begin{array}{l}d \\ j\end{array}\right) \beta_{j-1}$.

We will omit some of the proofs along the way, but refer to our paper Browder and Klee (2013) for further details.

To prove Theorem 3.1 it suffices to construct, for any $\beta_{i}$ and $h_{i}^{\prime}$ satisfying the conditions of the theorem, a Buchsbaum simplicial poset having those Betti numbers and $h^{\prime}$-numbers. Novik and Swartz (2009) showed it is sufficient to construct a family of Buchsbaum simplicial posets $X(k, d)$ for all $d$ and all $0 \leq k \leq d-1$ such that

$$
\tilde{\beta}_{i}(X(k, d))=\left\{\begin{array}{ll}
1, & \text { if } i=k \\
0, & \text { if } i \neq k,
\end{array} \text { and } \quad h_{j}^{\prime}(X(k, d))= \begin{cases}\left(\begin{array}{l}
d \\
j
\end{array}\right), & \text { if } j=0, k+1 \\
0, & \text { otherwise. }\end{cases}\right.
$$

It suffices to construct these complexes $X(k, d)$ for the following reason. Suppose we are given a sequence of Betti numbers and $h^{\prime}$-numbers satisfying the conditions of Theorem 3.1. The operation of gluing two disjoint simplicial posets together along a common facet is additive on both the $h^{\prime}$-numbers (with the exception of $h_{0}^{\prime}$ ) and Betti numbers, and it preserves the Buchsbaum property. Thus, we can construct a simplicial poset $P$ with the prescribed Betti numbers such that $h_{j}^{\prime}(P)=\left(\begin{array}{l}d \\ j\end{array}\right) \tilde{\beta}_{j-1}(P)$ for all $j$ by gluing together an appropriate selection of disjoint $X(k, d)$ posets. In order to extend this construction to allow for the cases that $h_{j}^{\prime}>\left(\begin{array}{c}d \\ j\end{array}\right) \tilde{\beta}_{j-1}$ for some values of $j$, we notice that the vector $\mathbf{h}=\left(h_{j}^{\prime}-\tilde{\beta}_{j}\right)_{j=0}^{d}$ has nonnegative entries, and hence it is the $h$-vector of some Cohen-Macaulay simplicial poset $Q$ by Theorem 2.2. Thus gluing $P$ to $Q$ along a facet will give the desired Buchsbaum simplicial poset. The precise details of this argument can be found in (Novik and Swartz, 2009, Lemma 7.8, Lemma 7.9).

For $X(0, d)$ we may take the disjoint union of two $(d-1)$-dimensional simplices, and for $X(d-$ $1, d)$ we may take two $(d-1)$-simplices identified along their boundaries. Novik and Swartz also gave constructions for $X(1, d)$ (Novik and Swartz, 2009, Lemma 7.6) and $X(d-2, d)$ (Novik and Swartz. 2009. Lemma 7.7) for all $d$, along with an ad-hoc construction for $X(2,5)$.

Our main result gives a unified construction of $X(k, d)$ for all $k$ and all $d$ that generalizes the known constructions when $k=0, k=1$, or $k=d-2$.

Theorem 3.2 For all $d \geq 2$ and all $0 \leq k \leq d-1$ there exists a Buchsbaum simplicial poset $X(k, d)$ with the following properties.

1. For all $0 \leq i \leq d-1$ and all $0 \leq j \leq d$,

$$
\tilde{\beta}_{i}(X(k, d))=\left\{\begin{array}{ll}
1, & \text { if } i=k \\
0, & \text { if } i \neq k,
\end{array} \quad \text { and } \quad h_{j}^{\prime}(X(k, d))= \begin{cases}\left(\begin{array}{l}
d \\
j
\end{array}\right), & \text { if } j=0, k+1 \\
0, & \text { otherwise. }\end{cases}\right.
$$

2. The link of each atom of $X(k, d)$ is shellable, and hence Cohen-Macaulay. 
3. For each atom $v$ of $X(k, d)$,

$$
h_{j}\left(\mathrm{l}_{X(k, d)}(v)\right)= \begin{cases}\left(\begin{array}{c}
d-1 \\
j
\end{array}\right), & \text { if } j=0, k \\
0, & \text { otherwise. }\end{cases}
$$

The familiar notion of shellability was extended to regular CW complexes by Björner (1984).

Definition 3.3 Let $\Delta$ be a pure $(d-1)$-dimensional regular $C W$-complex. For $\sigma$ a cell of $\Delta$, let $\partial \sigma$ denote the subcomplex consisting of all the proper faces of $\sigma$. A linear ordering $\sigma_{1}, \sigma_{2}, \ldots, \sigma_{r}$ of the maximal cells of $\Delta$ is called a shelling (and say we say $\Delta$ is shellable) if either $d=1$ or $d>1$ and the following are satisfied:

1. For $2 \leq j \leq r,\left(\bigcup_{i=1}^{j-1} \partial \sigma_{i}\right) \cap \partial \sigma_{j}$ is pure of dimension $d-2$,

2. there is a shelling of $\partial \sigma_{j}$ in which the $(d-2)$-cells of $\left(\bigcup_{i=1}^{j-1} \partial \sigma_{i}\right) \cap \partial \sigma_{j}$ come first for $2 \leq j \leq r$, and

3. $\partial \sigma_{1}$ is shellable.

Note that by Björner (1984), the order complex of the face poset of a shellable CW-complex is shellable (in the usual sense) and thus is Cohen-Macaulay. Furthermore, if $\Delta$ is a simplicial cell complex, then conditions (2) and (3) are satisfied automatically, as any ordering of the facets of the boundary of a simplex is a shelling order. Thus the definition of shellability for simplicial posets is completely analogous the the familiar notion of shellability for simplicial complexes.

To construct the posets $X(k, d)$, we adopt a technique that is related to the theory of manifold crystallizations, which will allow us to present a simplicial poset in terms of an edge-labeled dual graph. Ferri et al. (1986) is a nice survey on the subject, which has also been used by Murai (2010) to characterize simplicial cell decompositions of sphere products and real projective planes. Our approach differs slightly in that we do not require that our simplicial posets are even pseudomanifolds.

Definition 3.4 Let $G$ be a finite connected multigraph whose edges are labeled by colors in $[d]$. For any $S \subseteq[d]$, let $G_{S}$ be the restriction of $G$ to the edges whose label belongs to $S$ (and keeping all vertices of $G$ ). We define a poset $P(G)$ as follows: the elements of $P(G)$ are pairs $(H, S)$, where $S \subset[d]$ and $H$ is a connected component of $G_{S}$, ordered by $(H, S) \leq\left(H^{\prime}, S^{\prime}\right)$ if $S^{\prime} \subseteq S$ and $H^{\prime}$ is a subgraph of $H$.

By a straightforward argument as in Murai (2010) one may show that $P(G)$ is a simplicial poset of rank $d$ (though that paper only considers 'admissible' graphs, that assumption is not needed here). It is also helpful to consider the interpretation of the graph in terms of the simplicial cell complex associated to $P(G)$.

The maximal elements of $P(G)$ are those of the form $(F, \emptyset)$, where $F$ is a single vertex of $G$. Thus the facets of $P(G)$ correspond to the vertices of $G$, and we will from now on refer to the facet $(F, \emptyset)$ as 'the facet $F^{\prime}$. The unique minimal element of $P(G)$ is $(G,[d])$, and the vertices (i.e. atoms) of $P(G)$ are pairs of the form $(H,[d] \backslash\{c\})$. We fix the coloring of the vertices of $P(G)$ so that $(H,[d] \backslash\{c\})$ receives color $c$. It follows immediately from the definitions that no face of $P(G)$ contains two vertices of the same color.

If $F$ is a facet of $P(G)$ and $S \subseteq[d]$, let $F_{S}=(H,[d] \backslash S)$, where $H$ is the connected component of $G_{[d] \backslash S}$ containing $F$. Then $F_{S}$ corresponds to the unique face of $F$ whose vertices have the colors in $S$. 
Taking all of this together, we see that the simplicial cell complex $P(G)$ is constructed from $G$ in the following way: start with a collection of $(d-1)$-simplices indexed by the vertices of $G$, and identify each of their vertex sets with $[d]$. If two vertices $v$ and $w$ in $G$ are connected by an edge labeled $c$, identify the face of the simplex $v$ labeled $[d] \backslash\{c\}$ with that of $w$ in the natural way. Then the resulting simplicial cell complex has face poset $P(G)$. We will use these two ways of viewing $P(G)$ interchangeably. In particular, notice that if $F$ and $F^{\prime}$ are facets of $P(G)$, then $F_{S}=F_{S}^{\prime}$ if and only if $F$ and $F^{\prime}$ are in the same connected component of $G_{[d] \backslash S}$; in other words, if there is a path from $F$ to $F^{\prime}$ in $G$ that uses no edges colored by $S$.

We will often construct $G$ so that $G_{[d] \backslash\{c\}}$ is connected for all $c \in[d]$. In this case $P(G)$ has exactly one vertex of each color, and we will identify the vertices of $P(G)$ with the elements of $[d]$.

The following results allow us to interpret the shellability of a simplicial poset in terms of its dual crystal graph. We will see in Proposition 3.7 that the restriction sets of the shelling, along with the $h$-numbers of the resulting simplicial poset, can also be interpreted directly from the given multigraph.

Lemma 3.5 Let $G$ be an edge-colored multigraph such that $G_{[d] \backslash c}$ is connected for each $c \in[d]$. Then for each $c \in[d], \operatorname{lk}_{P(G)}(c)=P\left(G_{[d] \backslash c}\right)$.

Definition 3.6 Let $G$ be a connected multigraph with edges labeled in $[d]$. A linear ordering, $F_{1}, F_{2}, \ldots, F_{r}$, of the vertices of $G$ is a graphical shelling if for each $i \geq 1$, the set

$$
\left\{S \subseteq[d] \text { : every path from } F_{i} \text { to } F_{j} \text { with } j<i \text { uses some edge with color in } S\right\}
$$

has a unique minimal element, which we will call $R\left(F_{i}\right)$. Note that $R\left(F_{1}\right)=\emptyset$.

Proposition 3.7 Let $G$ be a connected multigraph with edges colored in $[d]$, and $F_{1}, F_{2}, \ldots, F_{r}$ a graphical shelling of $G$. Then the corresponding ordering $F_{1}, F_{2}, \ldots, F_{r}$ of the facets of $P(G)$ is a shelling. Furthermore, $h_{j}(P)=\left|\left\{i:\left|R\left(F_{i}\right)\right|=j\right\}\right|$.

\subsection{Constructing $X(k, d)$}

Now we are prepared to describe our construction of the simplicial posets $X(k, d)$ for all $d \geq 2$ and all $0 \leq k \leq d-2$. The graphs we will use to realize $X(k, d)$ will be defined in terms of binary words; the idea is somewhat reminiscent of the construction of the simplicial complexes $\mathcal{B}(i, d)$ in Klee and Novik (2012). Since $X(0, d)$ is the disjoint union of two $(d-1)$-simplices, we will only consider the simplicial posets $X(k, d)$ with $0<k<d$ for the remainder of this paper.

Definition 3.8 Suppose $k, d$ are positive integers with $k<d$. Let $W_{d}$ be the set of words on alphabet $\{0,1\}$ of length $d$ and first letter 1 . For $w=w_{1} w_{2} \ldots w_{d}$ in $W_{d}$, we may write $w$ as a concatenation of subwords $w=B_{1}(w) B_{2}(w) \ldots B_{q}(w)$ where $B_{i}(w)$ is a word of all 1's if $i$ is odd and all 0 's if $i$ is even. We will call these the blocks of $w$. For any $1 \leq j \leq d$, let $b_{j}(w)$ be the index of the block of $w$ containing $w_{j}$. Let $W_{d}(k)$ denote the set of words in $W_{d}$ with exactly $k+1$ blocks.

Definition 3.9 For $w=w_{1} w_{2} \ldots w_{d} \in W_{d}$ let $\operatorname{LEP}(w)=\left\{i>1: w_{i} \neq w_{i-1}\right\}$ be the set of left endpoints of $w$, and $\operatorname{REP}(w)=\left\{i<d: w_{i} \neq w_{i+1}\right\}$ be the set of right endpoints of $w$.

The terminology refers to the fact that $L E P$ and $R E P$ contain the indices of the first and last elements respectively of the blocks of $w$. Note that we do not include 1 as a left endpoint or $d$ as a right endpoint. However we allow 1 to be a right endpoint (if the first block has size one), and $d$ to be a left endpoint (if the last block has size one). 
Definition 3.10 For $k, d$ positive integers, $k<d$, let $G^{\prime}(k, d)$ be the edge-labeled multigraph on vertex set $W_{d}(k)$ such that if two words differ only in position $j$ they are connected by an edge labeled $j$.

Let $G(k, d)$ be the graph obtained by adding a new vertex, $\alpha$, to $G^{\prime}(k, d)$, and connecting a vertex $w=w_{1} w_{2} \ldots w_{d}$ of $G^{\prime}(k, d)$ to $\alpha$ by an edge labeled $j$ if $w_{j}$ is contained in a block of size one in $w$.

Example 3.11 The vertices of $G^{\prime}(1, d)$ correspond to binary words whose first letter is 1 that have two blocks. We label the vertices as

$$
\begin{aligned}
v_{1} & =100 \cdots 00 \\
v_{2} & =110 \cdots 00 \\
v_{3} & =111 \cdots 00 \\
& \vdots \\
v_{d-1} & =111 \cdots 10
\end{aligned}
$$

Thus the graph $G(1, d)$ is

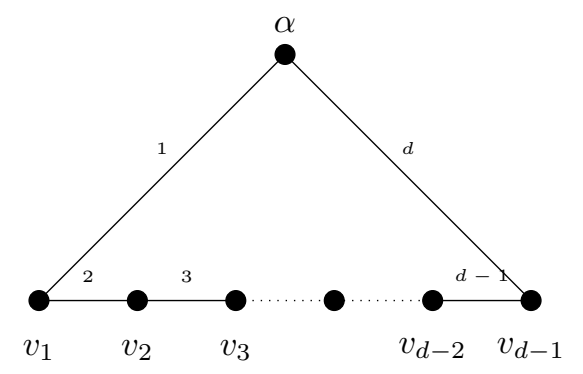

The corresponding simplicial poset $P(G(1, d))$ has $d$ facets, $F_{0}, F_{1}, F_{2}, \ldots, F_{d-1}$, where $F_{i}$ intersects $F_{i+1}$ along the facet opposite vertex $i+1$ for all $1 \leq i \leq d$ (where addition is taken modulo $d$ ). In this picture, $F_{0}$ corresponds to vertex $\alpha$, and $F_{j}$ corresponds to $v_{j}$ for all other $j$. This matches the complex $X(1, d)$ constructed by (Novik and Swartz, 2009. Lemma 7.6).

For concreteness, the geometric realization of $P(G(1,3))$ (a Möbius strip) is shown below with its underlying dual graph shown in gray.

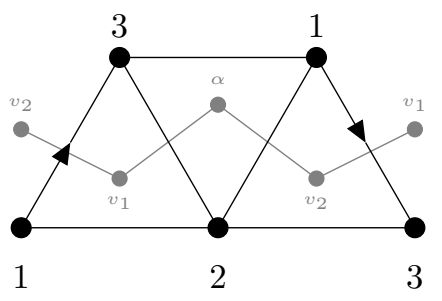

The simplicial cell complex $X(1, d)$ is either a trivial disc bundle (when d is even) or a twisted disc bundle (when $d$ is odd) over $\mathbb{S}^{1}$. In general, the complexes $X(k, d)$ are not pseudomanifolds (see Theorem 3.13 . 
Example 3.12 We will also illustrate $G(2,5)$. The vertices of $G(2,5)$ correspond to binary words whose first letter is 1 that have three blocks. The vertices in this case are

$$
\begin{array}{ll}
v_{12}=10111 & v_{23}=11011 \\
v_{13}=10011 & v_{24}=11001 \\
v_{14}=10001 & v_{34}=11101 .
\end{array}
$$

The graph $G(2,5)$ is shown below. We have drawn the vertex $\alpha$ three times to make the drawing planar, and we have made this vertex white in order to distinguish it from the vertices of $G^{\prime}(2,5)$.

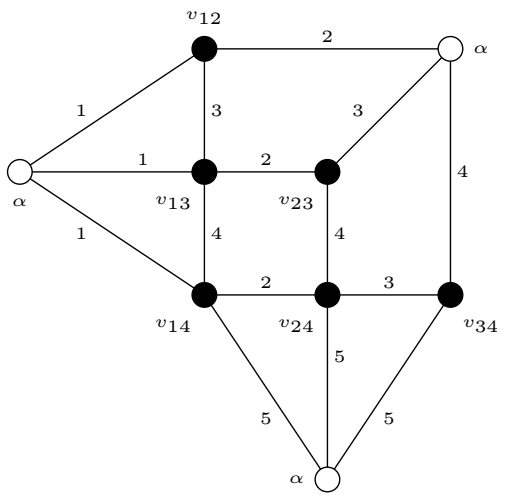

In this example, we see that the vertices $v_{12}, v_{13}$, and $v_{14}$ are all connected to vertex $\alpha$ by an edge labeled 1 . This means that the corresponding facets in $X(2,5)$ all intersect along a common ridge. Since $X(2,5)$ has a ridge that is contained in four facets, it cannot be a pseudomanifold. Novik and Swartz (2009) give an alternate construction of $X(2,5)$ that is obtained by removing the interior of one facet from a simplicial cell decomposition of $\mathbb{C P}^{2}$. Thus $X(2,5)$ can be realized as a manifold with boundary, even though we do not see this in our construction.

The proof of Theorem 3.2 has three main ingredients. We will only sketch the proof here, but complete details can be found in our full paper Browder and Klee (2013).

Proof: (Theorem 3.2)

1. We begin by showing (Browder and Klee, 2013, Lemma 3.1) that if $P$ is any Buchsbaum simplicial poset such that $f_{0}(P)=d$ and such that condition (3) of the theorem holds, then condition (1) of the theorem will also hold. This is the result of a straightforward inductive argument, but it is an important result because it allows us to conclude that $X(k, d)$ has the required Betti numbers and $h^{\prime}$-numbers without having to explicitly compute its homology groups.

2. The crux of the argument comes from showing that the link of each atom in $X(k, d)$ is shellable. We are able to give an explicit shelling order for the link of each atom (Browder and Klee, 2013, Theorem 4.13) because $X(k, d)$ only has $d$ atoms, and consequently each atom is contained in every facet.

The shelling of each link is obtained by taking facet $\alpha$ to be the first facet in the shelling order, followed by a lexicographic ordering on the remaining facets according to the positions where the 
corresponding binary word switches between blocks of zeros and blocks of ones (or vice-versa). A subtle point arises that we cannot just take the standard ordering on the ground elements $1,2, \ldots, d$, but rather that we must modify this ordering for each atom: for atom $i$, we order the ground set by declaring that $1<2<\cdots<i<d<d-1<\cdots<i+1$.

We are able to verify that this is a shelling order directly in terms of the multigraph $G(k, d)$ by using our definition of a graphic shelling.

3. The computation of the $h$-numbers of the link of each atom follows directly from our proof that the link of atom is shellable. We are able to explicitly compute the restriction set $R(F)$ for each facet in terms of the multigraph data.

As we have already mentioned, it is interesting to ask when a simplicial cell complex with the same $h^{\prime}$ numbers and Betti numbers as $X(k, d)$ can be realized as a simplicial cell manifold (with boundary). We have seen that our simplicial posets $X(1, d)$ always triangulate disk bundles over $\mathbb{S}^{1}$, and that our poset $X(2,5)$ is not even a pseudomanifold, but that $X(2,5)$ admits an alternate realization as a manifold with boundary. We show that when $\frac{d}{2} \leq k<d-1$, it is impossible to realize $X(k, d)$ as a pseudomanifold.

Theorem 3.13 Let $P$ be a $(d-1)$-dimensional Buchsbaum simplicial poset, $d>4$, satisfying condition (1) of Theorem 3.2 for some $k, \frac{d}{2} \leq k<d-1$. Then $P$ has a $(d-2)$-face that is contained in at least three $(d-1)$-faces.

It is an interesting question to investigate if $X(k, d)$ can be realized as a manifold when $1<k<\frac{d}{2}$.

\section{Acknowledgements}

We are grateful to Isabella Novik for numerous helpful conversations during the development of the ideas in this paper, and to Ed Swartz for suggesting the problem when $X(k, d)$ can be realized as a manifold.

\section{References}

L. J. Billera and C. W. Lee. A proof of the sufficiency of McMullen's conditions for $f$-vectors of simplicial convex polytopes. J. Combin. Theory Ser. A, 31(3):237-255, 1981.

A. Björner. Posets, regular CW complexes and Bruhat order. European J. Combin., 5(1):7-16, 1984. ISSN 0195-6698.

J. Browder and S. Klee. A classification of the face numbers of Buchsbaum simplicial posets. Math. Z., 2013. to appear. Preprint available: http: / / arxiv.org/abs/1307.1548.

M. Ferri, C. Gagliardi, and L. Grasselli. A graph-theoretical representation of PL-manifolds-a survey on crystallizations. Aequationes Math., 31(2-3):121-141, 1986. ISSN 0001-9054. doi: 10.1007/ BF02188181. URL http://dx.doi.org/10.1007/BF02188181.

G. Katona. A theorem of finite sets. In Theory of graphs (Proc. Colloq., Tihany, 1966), pages 187-207. Academic Press, New York, 1968. 
S. Klee and I. Novik. Centrally symmetric manifolds with few vertices. Adv. Math., 229(1):487-500, 2012. ISSN 0001-8708. doi: 10.1016/j.aim.2011.07.024. URL/http://dx.doi.org/10.1016/ j.aim.2011.07.024.

J. Kruskal. The number of simplices in a complex. In Mathematical optimization techniques, pages 251-278. Univ. of California Press, Berkeley, Calif., 1963.

F. Macaulay. Some properties of enumeration in the theory of modular systems. Proc. London Math. Soc., 26:531-555, 1972.

M. Masuda. $h$-vectors of Gorenstein* simplicial posets. Adv. Math., 194(2):332-344, 2005. ISSN 00018708. doi: 10.1016/j.aim.2004.06.009. URL http://dx.doi.org/10.1016/j.aim.2004. 06.009 .

S. Murai. Face vectors of simplicial cell decompositions of manifolds. Israel J. Math., 2010. To appear.

I. Novik and E. Swartz. Socles of Buchsbaum modules, complexes and posets. Adv. Math., 222(6):20592084, 2009. ISSN 0001-8708. doi: 10.1016/j.aim.2009.07.001. URL http://dx.doi.org/10 . $1016 / j . a i m .2009 .07 .001$

G. Reisner. Cohen-Macaulay quotients of polynomial rings. Adv. Math., 21(1):30-49, 1976. ISSN 00018708.

P. Schenzel. On the number of faces of simplicial complexes and the purity of Frobenius. Math. Z., 178 (1):125-142, 1981. ISSN 0025-5874. doi: 10.1007/BF01218376. URL http://dx.doi.org/ $10.1007 / \mathrm{BF} 01218376$

R. P. Stanley. Cohen-Macaulay complexes. In Higher combinatorics (Proc. NATO Advanced Study Inst., Berlin, 1976), pages 51-62. NATO Adv. Study Inst. Ser., Ser. C: Math. and Phys. Sci., 31. Reidel, Dordrecht, 1977.

R. P. Stanley. The number of faces of a simplicial convex polytope. Adv. in Math., 35(3):236-238, 1980. ISSN 0001-8708.

R. P. Stanley. $f$-vectors and $h$-vectors of simplicial posets. J. Pure Appl. Algebra, 71(2-3):319-331, 1991. ISSN 0022-4049. doi: 10.1016/0022-4049(91)90155-U. URL http://dx.doi.org/10.1016/ 0022-4049(91)90155-U.

R. P. Stanley. Combinatorics and commutative algebra, volume 41 of Progress in Mathematics. Birkhäuser Boston Inc., Boston, MA, second edition, 1996. ISBN 0-8176-3836-9.

E. Swartz. Lower bounds for $h$-vectors of $k$-CM, independence, and broken circuit complexes. SIAM J. Discrete Math., 18(3):647-661, 2004/05. ISSN 0895-4801. doi: 10.1137/S0895480102411358. URL http://dx.doi.org/10.1137/S0895480102411358 